\title{
Mesa inclinada en pacientes pediátricos con reto farmacológico, ¿es seguro?
}

\author{
Pediatrics patients head-up tilt test with pharmacological challenge, it is safe?
}

\author{
Alan Garcia ${ }^{1}$, Gabriela A. Bustillos-Garcia ${ }^{1 *}$ y Leonardo Rivera-Rodríguez ${ }^{2}$ \\ ${ }^{1}$ Departamento de Electrofisiología; ${ }^{2}$ Departamento de Cardiología Pediátrica. Instituto Nacional de Cardiología Ignacio Chávez, Ciudad de \\ México, México
}

\begin{abstract}
Resumen
El síncope en edades pediátricas representa una causa importante en las visitas a unidades de urgencias, por lo que excluir un origen cardíaco o maligno es fundamental al momento del abordaje inicial para determinar la conducta a seguir o la necesidad de derivar al cardiólogo pediatra o electrofisiólogo. El síncope vasovagal (SVV) es la causa más frecuente de síncope en pediatría, para cuyo diagnóstico basta una historia clínica detallada. Cuando ésta no es suficiente para determinar el diagnóstico de síncope reflejo o es necesario definir el tipo de respuesta que lo origina, está indicada una prueba de mesa inclinada que produce un estrés ortostático por la angulación y ello desencadena un síncope (fase pasiva). En pruebas no concluyentes está indicado un reto farmacológico para precipitar la respuesta hemodinámica, pero aún es un tema de controversia en edades pediátricas. El reto farmacológico incrementa la sensibilidad de la prueba, con una ligera reducción de la especificidad. Si bien no existe todavía un medicamento específico para la población pediátrica, los más empleados son los nitratos y el isoproterenol, este último relacionado con un mayor número de efectos adversos. La administración sublingual de los nitratos utilizados ha demostrado ser ideal, efectiva y segura en los pacientes pediátricos. El objetivo del artículo es realizar una revisión de las publicaciones médicas que demuestran la efectividad y seguridad del reto farmacológico durante la prueba de mesa inclinada en pacientes pediátricos, con énfasis en un estudio conducido en el Instituto Nacional de Cardiología con dinitrato de isosorbida (DNIS).
\end{abstract}

Palabras clave: Dinitrato de isosorbida (DNIS). Prueba de mesa inclinada. Síncope.

\begin{abstract}
Syncope in pediatrics represents an important cause of visits to the emergency units. For this reason, excluding a cardiac or malignant origin is essential at the time of the initial approach in order to determine what is the next step in management, or if they need to be referred to a pediatric cardiologist and/or electrophysiologist. Vasovagal syncope is the most frequent
\end{abstract}

\section{Correspondencia:}

*Gabriela A. Bustillos-García

Departamento de Electrofisiología

Instituto Nacional de Cardiología Ignacio Chávez

Juan Badiano, No. 1

Col. Belisario Domínguez Sección XVI, Del. Tlalpan

Fecha de recepción: 18-06-2019

Fecha de aceptación: 21-10-2019

C.P. 14080, Ciudad de México, México.

E-mail: gabustillosg@gmail.com

DOI: 10.24875/ACM. 19000260
Disponible en internet: 00-00-2000 Arch Cardiol Mex. 2020;90(2):178-188 www.archivoscardiologia.com 1405-9940/@ 2019 Instituto Nacional de Cardiología Ignacio Chávez. Publicado por Permanyer. Este es un artículo open access bajo la licencia CC BY-NC-ND (http://creativecommons.org/licenses/by-nc-nd/4.0/). 
cause of syncope in pediatrics, in which a detailed clinical history is enough to make the diagnosis. If no diagnosis is concluded by the history, or if it is necessary to define the hemodynamic response of the patients, the head-up tilt test is indicated; this will trigger syncope due to an orthostatic stress caused by the angulated table (passive phase). If a negative response remains, it can be followed by a pharmacologic challenge in order to trigger the hemodynamic response, which is still controversial in pediatrics. The pharmacologic challenge increases the sensitivity with a slight reduction in test specificity. Although there is not a specific drug for the challenge in pediatric patients yet, the most commonly drugs used are nitrates and isoproterenol, the latter related to a great number of adverse effects. Sublingual administration of nitrates in the challenge has been proven to be ideal, effective and safe in this specific age group. The aim of this article is to make a literature search in order to demonstrate the effectiveness and safety of the pharmacologic challenge during the head-up tilt test in pediatrics, emphasizing a study conducted at the National Institute of Cardiology with isosorbide dinitrate.

Key words: Head-up tilt test (tilt-test). Isosorbide dinitrate. Syncope.

\section{Introducción}

El síncope es un problema común en pediatría y representa el $1 \%$ de las consultas en el Servicio de Urgencias Pediátricas ${ }^{1}$. Se calcula una incidencia anual de 1.25 por cada 1,000 pacientes pediátricos y es más frecuente en mayores de $10 a^{n} 0 s^{2}$. El síncope recurrente puede afectar de forma notoria la calidad de vida del paciente debido a la etapa del desarrollo en la que se encuentra.

En general, el síncope se define como la pérdida transitoria de la conciencia secundaria a una hipoperfusión cerebral, se caracteriza por su naturaleza súbita y en ocasiones lo preceden pródromos de corta duración (síntomas de presíncope), casi siempre con una recuperación espontánea y completa sin estados de confusión posteriores al suceso. Sin embargo, la hipoperfusión cerebral puede generar movimientos similares a las convulsiones, sobre todo de tipo tónico-clónico o mioclónico, seguidos de períodos cortos de amnesia; esto puede confundir al médico durante el interrogatorio, en particular cuando el episodio no lo presenció otra persona que ayude a esclarecer el escenario clínico del síncope ${ }^{3}$ (Fig. 1).

Por otro lado, el presíncope se define como la sensación de pérdida del estado de conciencia sin llegar a ella y se caracteriza por síntomas secundarios tanto de hipoperfusión como de estimulación parasimpática, como sudoración, palidez, náusea, vómito, visión borrosa, entre otros ${ }^{4}$. Si bien la definición de síncope no está establecida con claridad cuando se habla de un origen neurocardiogénico secundario a una disfunción transitoria del sistema nervioso autónomo que ocurre en la población pediátrica, aún existen varios términos para referirse a él, entre ellos "desmayo", "síncope vasovagal", "síncope reflejo", "síncope neurocardiogénico".

De las causas de síncope, la vasovagal afecta a 15\% a $25 \%$ de los niños y adolescentes y es la causa más común de síncope en la población pediátrica $(61-80 \%$ de los $\left.\operatorname{casos}^{5,6}\right)$.

Debido a la complejidad del síncope, es fundamental obtener historias clínicas detalladas durante el abordaje inicial del paciente, que puede ser diagnóstico para síncope vasovagal sin requerir otro estudio complementario. De igual forma, es necesario un abordaje extenso que permita descartar otras causas como el origen primario, incluidas enfermedades cardiológicas de tipos obstructivo o arritmogénico, enfermedades neurológicas y metabólicas ${ }^{7,8}$ (Tabla 1).

Cuando existe duda diagnóstica y antes se han descartado otras causas de síncope, es preciso realizar una prueba de mesa inclinada para corroborar el origen vasovagal mediante una respuesta vasodepresora o cardioinhibitoria ${ }^{2,9}$.

\section{Prueba de mesa inclinada}

La prueba de inclinación es una herramienta útil, de bajo costo, que permite establecer el diagnóstico de síncope reflejo cuando no es seguro. A pesar de que la mayoría de los estudios se realiza en adultos, se ha documentado su uso seguro en la población pediátrica ${ }^{10}$. Dicha prueba no debe utilizarse con el fin de inducir o evaluar la respuesta al tratamiento farmacológico o intervencionista al paciente con SVV debido a la baja reproducibilidad de la prueba para desencadenar la misma respuesta hemodinámica en una segunda prueba ${ }^{2,6}$.

De manera inicial, la prueba de inclinación sólo incluía una fase pasiva y el estrés ortostático se desencadenaba debido a la inclinación de la mesa. Con posterioridad se empleó el llamado "método de los síntomas", que a través de un masaje carotídeo incrementaba el tono vagal hasta culminar en el síncope secundario a la respuesta vasodrepresora o cardioinhibitoria ${ }^{11}$. Por último se desarrolló la fase de provocación o reto farmacológico y el "protocolo italiano" es 


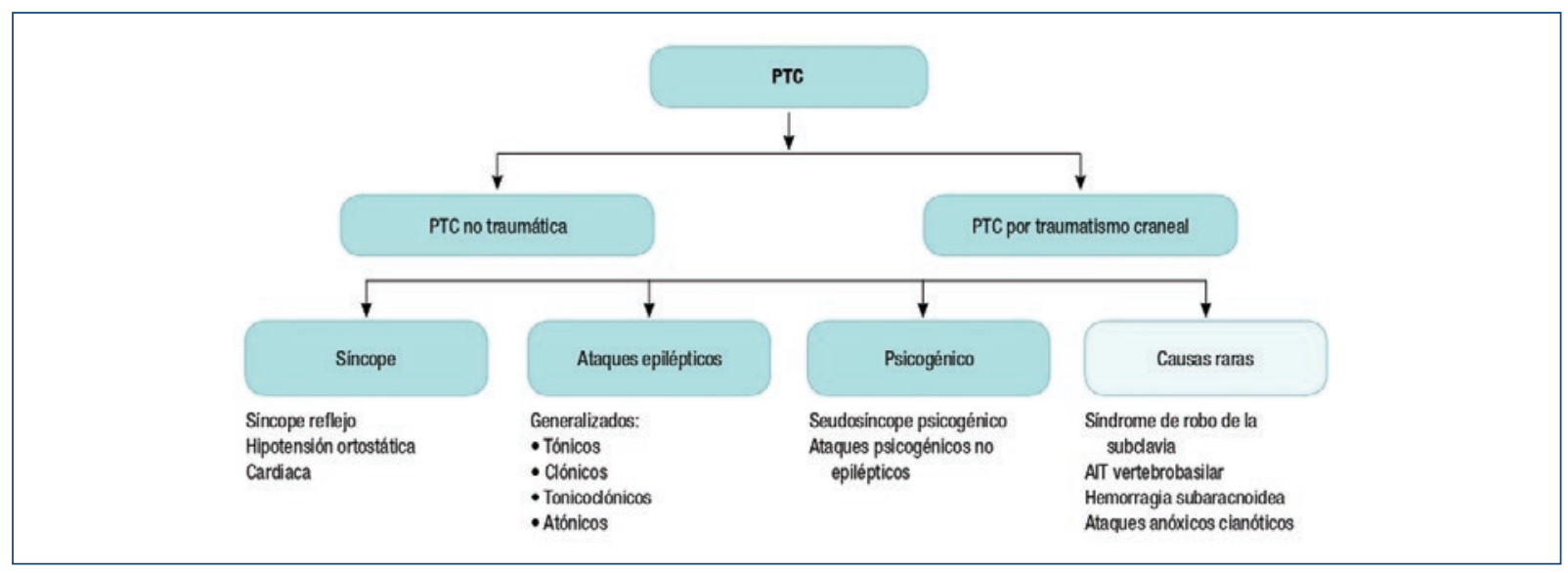

Figura 1. En el contexto de pérdida de conciencia no traumática, el trastorno se clasifica en cuatro grupos: síncope, crisis epilépticas, pérdida de conciencia transitoria psicógena y orígenes diversos (causas raras). La pérdida de conciencia no traumática puede causar caída con un traumatismo posterior a ésta, en cuyo caso la pérdida de la conciencia es traumática y no traumática. (Con autorización del Consenso de Síncope ESC 2018).

AIT: accidente isquémico transitorio; PTC: pérdida transitoria del conocimiento.

uno de los principales: utiliza trinitrato de glicerol (TNG) para demostrar una respuesta positiva para síncope con mayor rapidez y acortar no sólo la duración de la prueba sino que también incrementaba su sensibilidad con una ligera reducción de la especificidad ${ }^{12}$.

Más adelante, distintos protocolos modificaron algunos factores como la duración de las fases, tanto la pasiva como el reto farmacológico, y utilizaron también fármacos diferentes en este último en busca de aumentar la sensibilidad y especificidad de la prueba ${ }^{13}$.

\section{Metodología de la prueba de mesa inclinada}

La prueba de inclinación debe realizarse en un ambiente cómodo, relajado y alejado del ruido para evitar falsos positivos y negativos. Antes de iniciar el procedimiento es importante conocer si el paciente es alérgico a los medicamentos utilizados en el reto farmacológico si es necesario. El paciente debe hallarse bajo vigilancia continua a través de un electrocardiograma antes de iniciar la prueba y valorar la respuesta cardioinhibitoria, ya que algunos pueden experimentar síncopes por la punción. Por otra parte, el pletismógrafo colocado en un dedo o tipo brazalete en las extremidades documenta el componente vasodrepresor.

La prueba de inclinación posee dos fases: en la primera, llamada pasiva (no provocada), se recomienda que la mesa se incline a un ángulo entre $60^{\circ}$ y $70^{\circ}$, debido a que se ha demostrado que ángulos más altos o bajos reducen la sensibilidad y especificidad de la prueba $^{3,14}$. Esta fase tiene una duración de 20 a
40 minutos, tiempo suficiente para provocar el estrés ortostático. La fase pasiva es todavía la de primera línea en la prueba de inclinación. Cuando ésta permanece negativa y aún se requiere concluir un diagnóstico se continúa una segunda fase con reto farmacológico (fase provocada) durante 10 a 20 minutos, utilizando diversos nitratos o isoproterenol en la gran mayoría de los protocolos.

En el Instituto Nacional de Cardiología (México), la prueba de inclinación se realiza a una angulación de $70^{\circ}$ en la fase pasiva durante 20 minutos, seguido de una fase provocativa cuando aún no se establece un diagnóstico, y se utilizan $5 \mathrm{mg}$ de dinitrato de isosorbida durante 12 minutos, lo que permite el tiempo suficiente para desencadenar el síncope.

La prueba se considera positiva cuando el paciente reproduce síntomas de síncope o se documenta una respuesta hipotensora, cardioinhibitoria o ambas, con retorno del paciente a una posición de Trendelenburg en un lapso de 10 a 15 segundos y una recuperación espontánea. Al final se clasifica de acuerdo con la respuesta hemodinámica predominante, ya sea vasodepresora, cardioinhibitoria o mixta (Tabla 2); asimismo, la prueba permite diagnosticar otras causas de síncope, como hipotensión ortostática $(\mathrm{HO})$ o síndrome de taquicardia postural ortostática (STPO). La clasificación confiere un significado clínico a la prueba de inclinación (Tabla 3), con una tasa de positividad variable (Fig. 2).

Si bien todavía no se cuenta con una norma de referencia para el diagnóstico de síncope, la prueba con mesa inclinada es aún el método más empleado para fines diagnósticos 3,15 . A pesar de ello, debido a que 
Tabla 1. Causas comunes de pérdida del estado de conciencia transitoria en niños

\begin{tabular}{|c|c|}
\hline $\begin{array}{l}\text { Causas del síncope } \\
\text { ordenadas por frecuencia }\end{array}$ & Ejemplo \\
\hline Síncope vasovagal & $\begin{array}{l}\text { Típico: síntomas de pródromo } \\
\text { Atípico: sin síntomas de pródromo }\end{array}$ \\
\hline $\begin{array}{l}\text { Períodos de contención } \\
\text { de la respiración } \\
\text { (breath-holding spell) }\end{array}$ & $\begin{array}{l}\text { Breves períodos en los que los } \\
\text { niños dejan de respirar con } \\
\text { duración hasta } 1 \text { minuto } \\
\text { secundarios a emociones, como } \\
\text { enojo, sorpresa o lesiones }\end{array}$ \\
\hline Cardiológicas & $\begin{array}{l}\text { Alteraciones eléctricas primarias } \\
\text { - Síndrome de OT largo } \\
\text { - Síndrome de OT corto } \\
\text { - Síndrome de Brugada } \\
\text { - Síndrome de } \\
\text { Wolff-Parkinson-White (WPW) } \\
\text { - Taquicardia ventricular } \\
\text { polimórfica catecolaminérgica } \\
\text { Alteraciones cardíacas } \\
\text { estructurales } \\
\text { - Miocardiopatía hipertrófica } \\
\text { Anomalías de las arterias } \\
\text { coronarias } \\
\text { - Displasia arritmogénica del } \\
\text { ventrículo derecho } \\
\text { - Estenosis valvular aórtica } \\
\text { - Miocardiopatía dilatada } \\
\text { - Hipertensión pulmonar } \\
\text { - Miocarditis aguda }\end{array}$ \\
\hline Neurológicas & $\begin{array}{l}\text { Convulsiones } \\
\text { - Síndrome de Panayiotopoulos } \\
\text { Episodios vasculares } \\
\text { - Fenómeno de secuestro } \\
\text { sanguíneo subclavio } \\
\text { - Insuficiencia vertebrobasilar } \\
\text { Alteración de la circulación del } \\
\text { líquido cefalorraquídeo } \\
\text { - Quiste coloide del tercer } \\
\text { ventrículo } \\
\text { - Tumores de la fosa posterior } \\
\text { Crisis de vértigo } \\
\text { Migraña basilar } \\
\text { Narcolepsia/cataplexia } \\
\text { Sangrado, deshidratación, } \\
\text { hipoglucemia, alteraciones } \\
\text { hidroelectrolíticas. } \\
\text { Síndrome de conversión, } \\
\text { somatización, síndrome de } \\
\text { Munchausen/fingir la } \\
\text { enfermedad (malingering) } \\
\text { Ansiedad y síndrome de } \\
\text { hiperventilación }\end{array}$ \\
\hline De origen desconocido & \\
\hline
\end{tabular}

habitualmente los estándares documentados de la prueba se realizan en pacientes adultos, no existe una metodología específica en los pacientes pediátricos. Se ha documentado que en estos últimos son suficientes períodos de 10 minutos en la prueba a una angulación de la mesa de $60^{\circ}$ a $70^{\circ}$ para precipitar el
Tabla 2. Tipos de respuesta hemodinámica en la mesa de inclinación

\begin{tabular}{|c|c|}
\hline $\begin{array}{l}\text { Tipo de respuesta en la } \\
\text { mesa de inclinación }\end{array}$ & Características de la respuesta \\
\hline Tipo 1 o mixta & $\begin{array}{l}\text { La frecuencia cardíaca cae al } \\
\text { tiempo en que ocurre el síncope, } \\
\text { pero no menos de } 40 \text { Ipm; la caída } \\
\text { dura menos de } 10 \text { segundos. La PA } \\
\text { desciende antes que la FC }\end{array}$ \\
\hline $\begin{array}{l}\text { Tipo } 2 \mathrm{~A} \text { (cardioinhibitorio } \\
\text { sin asistolia) }\end{array}$ & $\begin{array}{l}\text { La frecuencia cardíaca cae } \\
<40 \text { lpm, por más de } 10 \text { segundos. } \\
\text { La PA desciende antes que la FC }\end{array}$ \\
\hline $\begin{array}{l}\text { Tipo 2B (cardioinhibitorio } \\
\text { con asistolia) }\end{array}$ & $\begin{array}{l}\text { Asistolia con duración }>3 \mathrm{~s} \text {. La } \\
\text { caída de la presión arterial coincide } \\
\text { o es posterior a la de la FC }\end{array}$ \\
\hline Tipo 3 o vasomotora & $\begin{array}{l}\text { La caída de la FC no excede más } \\
\text { del } 10 \% \text { en comparación con el } \\
\text { pico al momento del síncope }\end{array}$ \\
\hline $\begin{array}{l}\text { Incompetencia } \\
\text { cronotrópica }\end{array}$ & $\begin{array}{l}\text { No hay aumento significativo de la } \\
\text { FC durante la inclinación (p. ej., } \\
\text { menos del } 10 \% \text { de la FC antes de la } \\
\text { inclinación) }\end{array}$ \\
\hline $\begin{array}{l}\text { Síndrome de taquicardia } \\
\text { postural } \\
\text { ortostática (STPO) }\end{array}$ & $\begin{array}{l}\text { El ejercicio aumenta la FC } \\
\text { (> } 130 \text { lpm), al inicio y durante la } \\
\text { inclinación, antes del síncope }\end{array}$ \\
\hline
\end{tabular}

FC: frecuencia cardíaca; Ipm: latidos por minuto; PA: presión arterial.

síncope y se ha demostrado una especificidad del $85 \% 16$.

\section{Indicaciones}

1. Confirmar el diagnóstico de síncope en pacientes en quienes no se ha confirmado o reproducir los síntomas referidos por el sujeto con el patrón hemodinámico determinado tras una evaluación inicial ${ }^{3}$.

2. Evaluar la falla autonómica causante de hipotensión ortostática, ya sea por sospecha o confirmación de alteraciones en la presión arterial durante la evaluación inicial ${ }^{3}$.

3. Confirmar de forma diagnóstica el síndrome de taquicardia postural ortostática ${ }^{3}$.

4. Diferenciar ante la sospecha clínica del seudosíncope psicogénico respecto de otra causa de origen sincopal ${ }^{3}$.

\section{Contraindicaciones}

Las contraindicaciones a la prueba son estenosis mitral grave, enfermedades que ocasionan obstrucción del tracto de salida del ventrículo izquierdo, enfermedad arterial cerebral y enfermedad coronaria grave ${ }^{17}$. 
Tabla 3. Interpretación clínica de la prueba de inclinación (advertencias respecto de la interpretación del resultado en la mesa inclinada)

\section{Significado clínico de acuerdo con el resultado en la mesa de inclinación}

- Una prueba de mesa inclinada negativa no excluye el diagnóstico de síncope reflejo

- A pesar de la sensibilidad y especificad de la prueba de inclinación respecto de la presencia de susceptibilidad a la hipotensión, que quizá no sólo esté presente en el síncope reflejo sino también en otras causas de síncope, incluidas las de origen cardíaco. El concepto de susceptibilidad a la hipotensión más que un diagnóstico tiene una utilidad clínica importante, debido a que su ausencia o presencia juegan un papel importante para dirigir el tratamiento hacia un marcapasos en paciente afectados con síncope reflejo y en el control de la hipotensión, que es más frecuente en los pacientes ancianos

- Una prueba cardioinhibitoria positiva en la mesa inclinada predice con alta probabilidad la presencia de síncope con asistolia espontánea; éste es un hallazgo importante debido a la implicación de considerar marcapasos en el tratamiento de éstos. La presencia de una respuesta vasopresora o una respuesta mixta no excluye que el paciente padezca asistolia durante períodos de síncope espontáneo

- La prueba de inclinación es útil para separar el síncope que presenta convulsiones de los paciente que experimentan movimientos anormales debido a la epilepsia

- La prueba de inclinación tiene valor para distinguir el síncope de pacientes que sólo sufren caídas

- La prueba de inclinación es útil para separar el síncope del seudosíncope psicógeno. En pacientes con sospecha de seudosíncope psicógeno, la prueba de inclinación se realiza en conjunto con un EEG para vigilar y confirmar el diagnóstico

- La prueba de mesa inclinada no debe utilizarse para evaluar la eficacia de los tratamientos

EEG: electroencefalograma

\section{Reto farmacológico en la mesa inclinada en pacientes pediátricos}

Uno de los temores al realizar la prueba de mesa inclinada en pacientes pediátricos han sido los resultados falsos positivos obtenidos en algunos casos, debido a la falta de cooperación del paciente, el temor ante la prueba, la separación de los padres al ingresarlo a la prueba que suscita ansiedad y llanto, así como el temor del médico a las reacciones secundarias por el fármaco y los períodos de asistolia que son tanto más alarmantes cuanto más prolongados sean. Aunque la duración de la asistolia no está relacionada con la gravedad de la enfermedad y la mayoría recupera el estado de alerta de forma espontánea tras descender

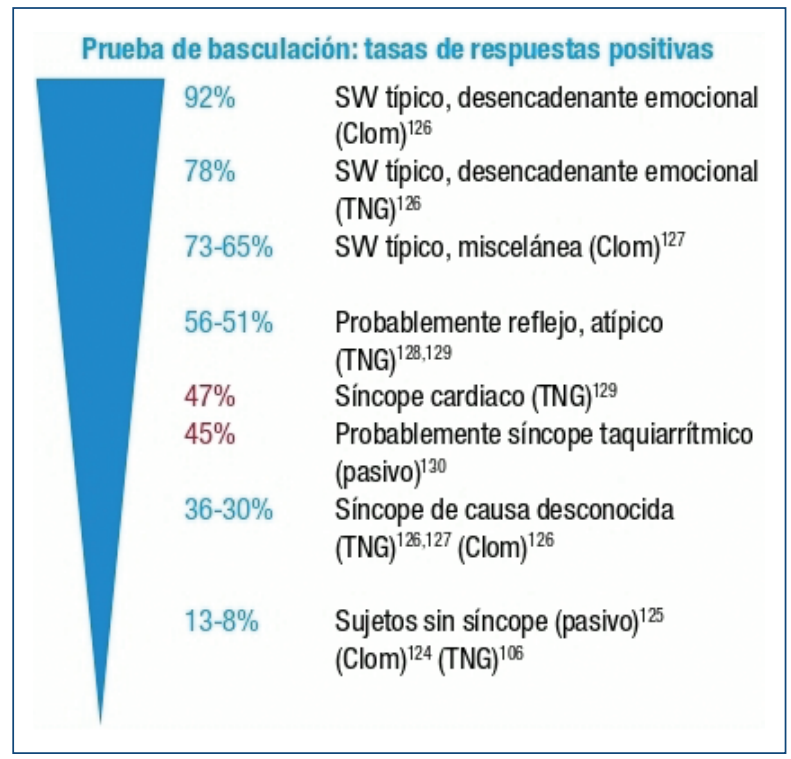

Figura 2. Tasa de positividad de la prueba de la mesa inclinada en diferentes condiciones clínicas. Estos estudios utilizaron el protocolo de Westminster para inclinación pasiva, el protocolo italiano para inclinación con trinitroglicerina y el protocolo de clomipramina por un total de 1,453 pacientes con síncope y 407 controles sin síncope. No se incluyeron los estudios que usan otros protocolos. (Con autorización del Consenso de Síncope ESC 2018)

Clom: clomipramina; TNG: trinitroglicerina; SVV: síncope vasovagal.

la cama a la posición supina, se han documentado episodios de paro cardiorrespiratorio secundarios a éstos ${ }^{18,19}$.

El reto farmacológico ha sido de gran utilidad y han incrementado la sensibilidad y especificidad de la prueba. Sin embargo, la controversia continúa respecto de cuál fármaco es de mayor utilidad para este grupo etario debido a la amplia variedad de resultados y los escasos estudios específicos que existen; el isoproterenol y los nitratos son los más utilizados.

\section{Isoproterenol}

La mayor parte de los efectos adversos en la prueba de inclinación con reto farmacológico se ha atribuido al uso del isoproterenol ${ }^{13,20}$. Se han notificado casos de infarto agudo al miocardio y arritmias ventriculares durante la administración de isoproterenol en el reto farmacológico; sin embargo, éstos se registraron en pacientes $>60$ años ${ }^{21}$. Otro punto relevante respecto de la vía de administración del isoproterenol es que la instrumentación intravenosa puede desencadenar síncope y ocasionar confusión en los resultados ${ }^{22}$. 
Lai, et al. llevaron a cabo un estudio en 79 pacientes pediátricos (intervalo de edad, 5.5-18 años), divididos en dos grupos, el primero con diagnóstico clínico de SVV (65 pacientes) y el segundo sin datos clínicos de SVV (14 pacientes). Ambos grupos se sometieron a una prueba con mesa inclinada, una primera fase sin reto farmacológico seguida de un reto con infusión de isoproterenol en los pacientes que durante la primera fase no desencadenaran síncope. Hasta 29 pacientes del grupo 1 y dos del grupo 2 requirieron un reto farmacológico para determinar el origen y el tipo de respuesta del síncope. El grupo 2 mostró diversos efectos adversos por el medicamente: dos pacientes sufrieron arritmias ventriculares, uno requirió marcapasos secundario a un bloqueo de segundo grado y el resto presentó extrasístoles ventriculares. Se concluyó que la infusión de isoproterenol incrementaba la sensibilidad de la prueba hasta un $45 \%$, y la especificidad decrece ligeramente de $93 \%$ a $86 \%{ }^{23}$. Sin embargo, la duración de la infusión del medicamento (30 min) y la dosis utilizada en este protocolo $(0.5-1 \mu \mathrm{g} / \mathrm{min})$ pudieron ocasionar un incremento de falsos positivos durante la prueba.

Se ha documentado que una menor duración de la infusión y dosis entre 0.5 y $5 \mu \mathrm{g} / \mathrm{min}$ del isoproterenol son suficientes para precipitar los síntomas, lo que evita así un mayor número de resultados erróneos ${ }^{24,25}$.

\section{Nitratos}

Los primeros estudios de Dindar, et al. demostraron un incremento de la sensibilidad de la prueba con el uso de DNIS en comparación con la fase pasiva sola con aumento de la sensibilidad de $77.5 \%$ respecto de $15 \%$ y un ligero cambio en la especificidad, $91.6 \%$ contra $100 \%$ respectivamente ${ }^{26}$. Con posterioridad, Karacan, et al., demostraron resultados similares en un estudio realizado en 29 pacientes en el que se comparó el DNIS con la prueba convencional sin reto farmacológico y se registró un incremento de la sensibilidad $(96.7 \%$ vs. $30 \%)$ con una ligera reducción de la sensibilidad ( $93.3 \%$ vs. $100 \%$, respectivamente), sin demostrar efectos adversos graves, de tal modo que puede indicarse como un método efectivo y seguro ${ }^{27}$.

\section{Estudios comparativos}

La sensibilidad (50-80\%) con el uso de la nitroglicerina resulta similar a la demostrada con la administración de isoproterenol (60-85\%) en diversos estudios. Sin embargo, a pesar de la reducción de la especificidad en el reto farmacológico, la especificidad es del 85-95\% con nitroglicerina en comparación con el isoproterenol, con una reducción de la especificidad de $35 \%$ a $83 \%$ 24,28-32.

A pesar de que Swissa, et al., en una comparación de 136 pacientes sometidos a reto farmacológico en la prueba de inclinación con isoproterenol, respecto de DNIS, demostró que el uso de este último ocasionaba un mayor número de bradiarritmias y duración de la respuesta asistólica, se notificó un solo caso de paro cardiorrespiratorio con recuperación tras maniobras de rescate ${ }^{33}$. Diversos estudios comparativos han demostrado un mayor número de respuestas positivas a la prueba hasta en un $12 \%$ con la nitroglicerina, así como una mejor tolerancia y un menor número de efectos adversos en comparación con el uso del isoproterenol $^{34,35}$. En otro estudio realizado en el Instituto Nacional de Cardiología en la Ciudad de México, que se describe a continuación, se demostró con una cohorte mayor de pacientes el uso seguro y efectivo de dicha prueba (se utilizó DNIS). De igual manera, el empleo sublingual de la nitroglicerina en la fase provocativa de la prueba, sobre todo en los pacientes pediátricos, evita que la administración intravenosa incremente tanto el estrés psicológico del paciente como los resultados falsos positivos.

\section{Estudio realizado en pacientes del instituto nacional de cardiología}

Se realizó un análisis retrospectivo de los resultados en las pruebas de mesa inclinada llevadas a cabo en el Instituto Nacional de Cardiología Ignacio Chávez en pacientes $\leq 18$ años con historia clínica indicativa de síncope vasovagal o de origen desconocido, entre 2015 y 2018. Se incluyó a 220 pacientes divididos por edades en dos grupos: grupo $1<10$ años (36 pacientes) y grupo 2 de 10 a 18 años (183 pacientes). No se observó un sexo predominante en ninguno de los dos grupos. En 179 pacientes (83\%) del total de la muestra fue necesario el reto farmacológico en la prueba con mesa inclinada para determinada su respuesta hemodinámica. La tasa de respuesta positiva del total de la muestra fue del $66 \%$ (grupo 1, 50\%; grupo 2, $70 \%$ ). En ambos grupos el patrón de respuesta hemodinámica que predominó fue el mixto (grupo 1, 50\%, grupo 2, 56\%).

Grupo I: 36 pacientes, con intervalo de edad de 6 a 10 años (media de 7.8). La relación en cuanto al sexo fue de 1.25:1 (20/16) en favor del sexo masculino. La principal indicación de la prueba fue el síncope en 23 pacientes, presíncope en 13 pacientes. La prueba fue 
positiva en 18 pacientes, 3 mostraron respuesta positiva durante la fase 1 (sin reto farmacológico) y el resto requirió la administración de DNIS. El tiempo de respuesta osciló entre 4 y 12 minutos con una media de 9.3. La respuesta hemodinámica predominante fue de tipo mixto en 9 pacientes (50\%), seguido por disautonomía (4) y respuesta cardioinhibitoria 2B (4) con pausas entre 3 y 6 segundos; sólo un paciente presentó respuesta cardioinhibitoria $2 \mathrm{~A}$.

Grupo II: 183 pacientes con edades entre 11 y 18 años (media, 14.8). No se observó diferencia de género (relación 1:1). La prueba fue positiva en 128 pacientes (70\%), 31 durante la fase 1 y el resto requirió la administración de DNIS sublingual. El tiempo de respuesta durante la prueba fue variable, de 1 a 20 minutos con una media de 8.5 minutos. La respuesta hemodinámica preponderante fue de tipo mixta en 43 pacientes (33.7\%), seguida por disautonomía en 32 pacientes (25\%), cardioinhibitoria $2 \mathrm{~B}$ en 25 pacientes (19.5\%), vasodepresora en 14 pacientes (11\%), cardioinhibitoria $2 A$ en 13 pacientes (10\%) y sólo un paciente presentó STPO (0.8\%).

En todos los casos de asistolia durante la prueba se logró la recuperación tras posicionar al paciente en decúbito supino y administrar fluidoterapia. No se registraron complicaciones durante el estudio. Esta prueba demuestra, al igual que otras ya mencionadas, el uso seguro del DNIS sublingual en paciente pediátricos.

\section{Tratamiento}

\section{Medidas no farmacológicas}

En niños con síncope vasovagal, las medidas preventivas son todavía la primera elección del tratamiento e incluyen tranquilizar al paciente (se le indica que se trata de un procedimiento benigno con la finalidad de reducir el estrés psicológico), evitar los factores desencadenantes de síncope e incrementar su ingestión de sal ( $2 \mathrm{~g}$ al día); los líquidos ( $\mathrm{L}$ diarios) reducen dichos episodios ${ }^{36}$. El entrenamiento mediante maniobras físicas que incrementan el retorno venoso y la tolerancia ortostática han demostrado ser efectivos para revertir el síncope ${ }^{37,38}$. Diversos estudios clínicos han probado la efectividad del tratamiento no farmacológico y reducido la recurrencia del síncope, de un 56\% en pacientes sin tratamiento a un $39 \%$ cuando se aumentaba el consumo de líquidos y sal $(P=0.029)^{39}$. Es fundamental enfatizar la disciplina en los cambios dietéticos (ingestión de agua y sal) y en el entrenamiento físico para prevenir el síncope en pacientes pediátricos. En fecha reciente se condujo un estudio transversal realizado en un total de 70 pacientes con edades de 5 a 20 años, divididos en dos grupos; el grupo 1 se conformó con 30 pacientes (13 masculinos/17 femeninos) que recibían medidas farmacológicas y no farmacológicas y se comparó con el grupo 2 , integrado con 40 pacientes (18 masculinos/22 femeninos) tratados sólo con medidas no farmacológicas (incremento de agua y sal en la dieta, maniobras físicas). Ambos grupos recibieron seguimiento por tres años y se observó una diferencia significativa en los síntomas de presíncope y síncope en los pacientes tratados sólo de manera no farmacológica (grupo 2), con una recurrencia a los tres años del $5 \%$ en comparación con los pacientes que combinan el mismo tratamiento con medidas farmacológicas (grupo 1), en los cuales la recurrencia del síncope fue de $44 \%(P=0.001)$; debe mencionarse que los resultados obtenidos pueden deberse a que los pacientes sometidos a los tratamientos farmacológico y no farmacológico tienden a reducir el seguimiento y uso correcto de este último; se concluye así que las medidas preventivas utilizadas de manera correcta son suficientes para el tratamiento de pacientes con síncope neurocardiogénico ${ }^{40}$.

\section{Medidas farmacológicas}

El uso de fármacos para el tratamiento del síncope vasovagal debe considerarse como segunda línea en los pacientes pediátricos, en quienes continúe la recurrencia del síncope a pesar del seguimiento correcto de las medidas preventivas. Entre los medicamentos más utilizados se encuentran los betabloqueadores, midodrina (agonista de receptores $\alpha$ ), fluorohidrocortisona y los inhibidores de la recaptura de serotonina; sin embargo, aún no se ha encontrado el fármaco ideal debido a la baja respuesta al tratamiento. Pese a ello, si bien existen pocos estudios del tratamiento con midodrina en pacientes pediátricos, han demostrado una gran efectividad en la reducción del síncope. Se efectuó un estudio aleatorizado en 26 pacientes con límites de edad de 6 a 16 años divididos en dos grupos, el grupo 1 con 13 pacientes tratados con midodrina y medidas no farmacológicas y el grupo 2 (13 pacientes) tratados sólo con medidas no farmacológicas, durante un seguimiento corto de seis meses. Se demostró una reducción de la recurrencia del síncope vasovagal resistente al tratamiento conservado, con una tasa de recurrencia del $80 \%$ en el grupo 1 en comparación con el $22 \%$ en los pacientes tratados con midodrina $(P=0.023)$. No se observó hipertensión supina en 
ninguno de los sujetos tratados con midodrina y sólo uno experimentó malestar gastrointestinal durante el tratamiento ${ }^{41}$. En un segundo estudio realizado en 48 pacientes con edades de 6 a 17 años con síncope o síntomas de presíncope, los participantes se asignaron a tres grupos de manera aleatorizada para comparar distintos tratamientos. El grupo 1 incluyó a individuos tratados con medidas no farmacológicas, el grupo 2 a sujetos con tratamiento a base de cresol (placebo) y el grupo 3 a enfermos tratados con midodrina, cada grupo conformado por 16 pacientes, durante un seguimiento aproximado de nueve meses. Se concluyó que la midodrina era efectividad para reducir el síncope cuando se comparaba con los otros tratamientos empleados $(P=0.05)^{42}$. A pesar de los resultados de ambos estudios, la duración del seguimiento tan corto, al igual que la evaluación de la respuesta al tratamiento mediante la repetición del estudio con mesa inclinada, limita los resultados obtenidos debido a que las guías mencionan la escasa utilidad de esta última para la evaluación terapéutica ${ }^{3}$. Otra posibilidad de la efectividad demostrada puede ser secundaria al tipo de respuesta hemodinámica predominante (vasodepresora), que se correlaciona con una mayor tasa de efectividad al utilizar un medicamento agonista de receptores $\alpha_{1}{ }^{41}$.

La evidencia del beneficio de la fluorohidrocortisona es limitada debido a que existen pocos estudios conducidos en este grupo etario. Se condujo un estudio aleatorizado, doble ciego, controlado con placebo, en 32 pacientes (uno perdido durante el estudio), todos < 18 años. El grupo 1 se integró con 18 pacientes tratados con complementos de sal y fluorohidrocortisona y el grupo 2 con placebo (14 pacientes), con un seguimiento aproximado de un año. Se encontró que, al contrario de la hipótesis planteada en favor del uso de la fluorohidrocortisona, presentaban una menor recurrencia del síncope en el grupo placebo $(P=0.04)$ y sólo el $53 \%$ del total de pacientes tratados no sufrió síncope durante el seguimiento; se observó por tanto una baja efectividad del fármaco empleado ${ }^{43}$. Estos resultados son similares a los de un estudio comparativo entre la fluorohidrocortisona ( $0.2 \mathrm{mg} / \mathrm{día}$ ) y atenolol (100 mg/día) para el tratamiento del síncope vasovagal, en el cual la tasa de recurrencia fue del 52\% (30 pacientes) del total de sujetos tratados en ambos grupos (total de 58 individuos) ${ }^{44}$.

Los betabloqueadores son todavía un tratamiento con recomendación de clase III (sin beneficios) para los pacientes pediátricos, según las guías para el tratamiento de síncope ${ }^{3,16}$, a pesar de que se demostró de manera inicial una reducción del síncope recurrente en un $60 \%$ en 21 pacientes tratados con metoprolol ${ }^{45}$. Más adelante, en un estudio prospectivo y aleatorizado, realizado en pacientes con edades de 8 a 17 años con un seguimiento durante un año, se demostró que el tratamiento con metoprolol se relacionaba con una mayor tasa de recurrencia de síncope respecto del tratamiento no farmacológico, $43 \%$ contra $29 \%$ respectivamente ( $P$ $=0.389)^{46}$. Sin embargo, en fecha reciente se efectuó un estudio de 38 pacientes pediátricos con síncope vasovagal, con edades de 6 a 13 años en quienes se realizó un análisis de los valores de NE en orina de 24 horas con el objetivo de demostrar que las cifras elevadas de NE en la orina están relacionadas con una mayor respuesta al tratamiento con metoprolol. Dichos pacientes se compararon al principio con un grupo control sano de 20 pacientes. Tras realizar una medición de los valores de NE en orina de 24 horas en pacientes con SVV (31.62 $\pm 14.11 \mu \mathrm{g} / 24 \mathrm{~h})$ se registró un coeficiente de dispersión muy elevado $\left(R^{2}=0.0028\right)$ comparado con el grupo control en el cual las cifras de NE en orina de 24 horas de $35.04 \pm 7.28 \mu \mathrm{g} / 24 \mathrm{~h}$ y que presentaba un bajo coeficiente de dispersión $\left(R^{2}=0.0002\right)$. Estos resultados demuestran las diferencias individuales entre los pacientes con SVV, en quienes la cuantificación de NE en la orina tiene un posible valor predictivo para la evaluación de un tratamiento. Debido a los resultados obtenidos, se evaluó el uso del metoprolol en los 38 pacientes enfermos y se concluyó que los pacientes que presentan una respuesta efectiva al metoprolol están ligados a cifras elevadas de NE en orina de 24 horas en comparación con los que no presentan respuesta al tratamiento $(40.75 \pm 12.86$ vs. $21.48 \pm 6.49$ respectivamente). De igual manera, se estableció que los pacientes con valores elevados de NE en orina de 24 horas tienen una elevación mayor de la presión arterial supina tanto sistólica como diastólica y que los valores de NE en orina de 24 horas $>34.84 \mu \mathrm{g} / 24 \mathrm{~h}$ son indicadores de que el tratamiento con metoprolol puede ser efectivo en pacientes pediátricos, con una especificidad del $100 \%$ y sensibilidad del $70 \%{ }^{47}$. Esto abre una puerta para estudios posteriores basados en los principios presentados en este estudio.

\section{Tratamientos intervencionistas}

El marcapasos en los pacientes pediátricos es todavía un tema controversial debido a la naturaleza benigna de la enfermedad e incluso existe en las guías aún controversia respecto de su uso. Las guías de la AHA/ACC/HRS (2017) para la evaluación y el tratamiento del síncope lo clasifican con nivel de evidencia 
Ilb, con base en dos estudios efectuados en 22 pacientes pediátricos, los cuales presentaban períodos de apnea que provocaban en ocasiones convulsiones secundarias a la anoxia cerebral y en quienes luego se documentaban períodos prolongados de asistolia (> $4 \mathrm{~s}$ ); tras la colocación del marcapasos se redujeron hasta un $86 \%$ los episodios de síncope ${ }^{16}$.

Aún no existe evidencia suficiente para considerar al marcapasos como una indicación absoluta cuando el paciente satisface dichos criterios clínicos, al igual que todavía no se ha estudiado qué tipo de marcapasos utilizar debido a que tanto el marcapasos de una cámara con histéresis como el de doble cámara demuestran una efectividad similar en la reducción del síncope ${ }^{48,49}$. Sin embargo, aún se requieren estudios que comparen ambos marcapasos, así como un seguimiento a largo plazo de estos pacientes.

Por otro lado, las guías de la European Society of Cardiology (ECS 2018) no recomiendan el uso de marcapasos en pacientes pediátricos ni tampoco en adultos jóvenes; los pacientes $>40$ años son los enfermos ideales cuando cumplen los criterios para el procedimiento ${ }^{3}$.

La cardioneuroablación se ha propuesto como un tratamiento novedoso para pacientes que presentan una respuesta de tipo cardioinhibitoria. Este tratamiento consiste en la ablación de plexos ganglionares parasimpáticos intracardíacos, localizados de forma predominante en la pared posterior de la aurícula izquierda. El objetivo es reducir el tono vagal y evitar así el componente cardioinhibitorio al aumentar la frecuencia cardíaca para reducir los episodios de síncope. Aunque no existen estudios de cohortes en pacientes pediátricos, algunos casos aislados como el de Suenega, et al. ${ }^{50}$ en una paciente de 17 años y el de Debruyne, et al. ${ }^{51}$ en un individuo de 16 años, demuestran resultados promisorios, en los cuales los pacientes estuvieron libres de síncope durante un seguimiento de 12 y 22 meses, respectivamente, tras la intervención. A pesar de los buenos resultados, se requieren estudios con cohortes más grandes para considerar la cardioneuroablación como una opción terapéutica para pacientes pediátricos.

\section{Conclusiones}

El síncope neurocardiogénico constituye un reto diagnóstico en pediatría. En la evaluación inicial es necesario descartar antecedentes cardiológicos personales y heredofamiliares en búsqueda de muerte súbita $y$, ante la sospecha o duda, es necesario referirlo al cardiólogo o electrofisiólogo pediatra una vez que se han descartado los problemas neurológicos y psicógenos como origen primario del síncope ${ }^{52}$.

La prueba con mesa inclinada ayuda a despejar la sospecha diagnóstica de síncope neurocardiogénico, que es la causa más frecuente de síncope. En los pacientes pediátricos aún existe controversia respecto del uso del reto farmacológico durante la prueba con mesa inclinada, pero diversos estudios han documentado el uso seguro de ésta a pesar de que se han documentado casos de paro cardiorrespiratorio en las pruebas de inclinación y otros efectos adversos atribuidos al uso del isoproterenol durante el reto farmacológico, si bien en su mayoría se trata de estudios realizados en adultos en quienes las comorbilidades juegan un papel importante. El uso de nitratos ha demostrado ser efectiva y segura en los pacientes pediátricos y su administración sublingual facilita su uso y beneficia la prueba, lo que evita resultados falsos positivos por el estrés secundario a la punción para la administración del medicamento. El temor del médico al uso de este compuesto limita los resultados de la prueba, dado que el reto farmacológico eleva la sensibilidad con una ligera reducción de la especificidad.

El tratamiento de primera elección son las medidas no farmacológicas, las cuales suponen educar al paciente y cambiar su dieta (incremento de agua y sal). El tratamiento farmacológico ha demostrado ser efectivo en pocos estudios, de modo específico en el caso de la midodrina. Aunque algunos estudios recientes demuestran que el metoprolol puede ser efectivo si se basa su tratamiento en la cuantificación de NE en orina de 24 horas, este estudio abre un campo en la investigación en el tratamiento farmacológico.

El uso de marcapasos para el tratamiento del síncope neurocardiogénico en los pacientes pediátricos aún es controversial incluso en las guías de síncope $e^{3,15}$ y la poca evidencia limita su uso al no tener criterios de implantación claramente establecidos. La cardioneuroablación resulta un tratamiento prometedor en pacientes que muestran respuesta cardioinhibitoria durante el síncope debido al éxito obtenido en los informes de caso aislados y ha demostrado ser efectiva, segura y sin recurrencia de síncope durante su seguimiento. Sin embargo, se requieren más estudios al respecto dirigidos a la población pediátrica.

\section{Financiamiento}

Esta investigación no ha recibido ninguna beca específica de agencias de los sectores públicos, comercial, o sin ánimo de lucro. 


\section{Conflicto de intereses}

Ninguno.

\section{Responsabilidades éticas}

Protección de personas y animales. Los autores declaran que para esta investigación no se han realizado experimentos en seres humanos ni en animales.

Confidencialidad de los datos. Los autores declaran que en este artículo no aparecen datos de pacientes.

Derecho a la privacidad y consentimiento informado. Los autores declaran que en este artículo no aparecen datos de pacientes.

\section{Bibliografía}

1. Driscoll DJ, Jacobsen SJ, Porter CJ, Wollan PC. Syncope in children and adolescents. J Am Coll Cardiol 1997;29:1039-45.

2. Lewis DA, Dhala A. Syncope in the pediatric patient. Pediatr Clin North Am 1999;46:205-19.

3. Brignole M, Moya A, Lange FJ, Deharo JC, Elliott PM, Fanciulli A, et al. ESC Guidelines for the diagnosis and management of syncope. Eur Heart J 2018;39:1883-1948.

4. Fish FA, Strasburger JF, Benson DW Jr. Reproducibility of symptomatic response to upright tilt in young patients with unexplained syncope. Am J Cardiol 1992;70:605-9.

5. Moodley M. Clinical approach to syncope in children. Semin Pediatr Neurol 2013;20:12-17.

6. Bayram AK, Pamukcu O, Per H. Current approaches to the clinical assessment of syncope in pediatric population. Childs Nerv Syst 2016;32:427-36.

7. Zhang Q, Du J, Wang C, Zhongdong D, Li W, Chaoshu T. The diagnostic protocol in children and adolescents with syncope: a multi-centre prospective study. Acta Pediatr 2009;98:879-84.

8. Rivera-Rodríguez L. El niño con síncope. Acta Pediatr Mex 2015;36: 352-60.

9. Zygmunt A, Stanczyk J. Heart rate variability in children with neurocardiogenic syncope. Clin Auton Res 2004;14:99-106.

10. Dalla PR, Kleinmann A, Zysk S, Bechtold S, Netz N. Head-up-tilt testing in children: new perspectives using beat-to-beat blood-pressure monitoring. Images Paediatr Cardiol 2005;7:1-7.

11. Puggioni E, Guiducci V, Brignole M, Menozzi C, Oddone D, Donateo P, et al. Results and complications of the carotid sinus massage performed according to the "method of symptoms". Am J Cardiol 2002;89:599-601.

12. Bartoletti A, Alboni $P$, Ammirati F, Brignole M, Del Rosso A, Foglia MG et al. "The Italian protocol": a simplified head-up tilt testing potentiated with oral nitroglycerin to assess patients with unexplained syncope. Europace 2000;2:339-42.

13. Parry SW, Reeve P, Lawson J, Shaw EF, Davison J, Norton M, et al. The Newcastle protocols 2008: an update on head-up tilt table testing and the management of vasovagal syncope and related disorders. Heart 2009:95:416-20.

14. Benditt DG, Ferguson DW, Grubb BP, Kapoor WN, Kungler J, Lerman BB. Tilt table testing for assessing syncope. J Am Coll Cardiol 1996;8: 263-75

15. Shen W-K, Sheldon RS, Benditt DG, Cohen MI, Forman DE, Goldberger ZD et al. 2017 ACC/AHA/HRS guideline for the evaluation and management of patients with syncope: a report of the American College of Cardiology/ American Heart Association Task Force on Clinical Practice Guidelines and the Heart Rhythm Society. Circulation 2017;136:e60-e122.

16. Vlahos AP, Tzoufi M, Katsouras CS, Barka T, Sionti I, Michalis LK, et al. Provocation of neurocardiogenic syncope during head-up tilt testing in children: comparison between isoproterenol and nitroglycerin. Pediatrics 2007;119:e419-25.

17. Kenny RA, O'Shea D, Parry SW. The Newcastle protocols for head-up tilt table testing in the diagnosis of vasovagal syncope, carotid sinus hypersensitivity, and related disorders. Heart 2000;83:564-9.

18. Raviele A. Tilt-induced asystole: a useful prognostic marker or clinically unrelevant finding? Eur Heart J 2002;23:433-7.

19. Maloney JD, Jaeger F, Fouad-Tarazi F, Morris $H$. Malignant vasovaga syncope: prolonged asystole provoked by head-up tilt. Cleve Clin J Med $1988 ; 55: 542-8$
20. Kim PH, Ahn SJ, Kim JS. Frequency of arrhythmic events during head-up tilt in patients with suspected neurocardiogenic syncope or presyncope. Am J Cardiol 2004;94:1491-5.

21. Wang $\mathrm{CH}$, Hung MJ, Kuo LT, Cherng WJ. Cardiopulmonary resuscitation during coronary vasospasm induced by tilt table testing. Pacing Clin Electrophysiol 2000;23:2138-40

22. Stevens PM. Cardiovascular dynamics during orthostatis and the influence of intravascular instrumentation. Am J Cardiol 1966;17:211-18.

23. Lai WT, Chen MR, Lin SM, Hwang HK. Application of head-up tilt table testing in children. J Formos Med Assoc 2010;109:641-6.

24. Shen WK, Jahangir A, Beinborn D, Lohse CM, Hodge DO, Rea RF, et al. Utility of a single stage isoproterenol tilt table test in adults. A randomized comparison with passive head-up tilt. J Am Coll Cardiol 1999;33: 985-90.

25. Cohen TJ, Chengot T, Chengot M, Catania S, Quan W. A comparison of a single-stage isoproterenol tilt table test protocol with conventional two-stage tilt protocol in patients with syncope. J Invasive Cardiol 2002;14:430-1.

26. Dindar A, Cetin B, Ertuğrul T, Cantez T. Sublingual isosorbide dinitrate stimulated tilt test for diagnosis of vasovagal syncope in children and adolescents. Pediatr Cardiol 2003;24:270-3

27. Karacan $\mathrm{M}$, Olgun $\mathrm{H}$, Ceviz $\mathrm{N}$. Head-Up tilt table testing with sublingual isosorbide dinitrate in the diagnosis of vasovagal syncope in children. Eurasian J Med 2008;40:115-18.

28. Saal DP, Thijs RD, van Dijk JG. Tilt table testing in neurology and clinical neurophysiology. Clin Neurophysiol 2016;127:1022-30.

29. Cheshire WP Jr. Syncope. Continuum (Minneap Minn) 2017;23:335-58.

30. Raviele A, Menozzi C, Brignole M, Gasparini G, Alboni P, Musso G, et al. Value of head-up tilt testing potentiated with sublingual nitroglycerin to assess the origin of unexplained syncope. Am J Cardiol 1995;76: 267-72.

31. Aerts AJ, Dendale P. Nitrate stimulated tilt table testing: a review of the literature. Pacing Clin Electrophysiol 2003;26:1528-37.

32. Aerts AJ, Dendale P, Block P, Dassen WR. Reproducibility of nitrate-stimulated tilt testing in patients with suspected vasovagal syncope and a healthy control group. Am Heart J 2005;150:251-6.

33. Swissa M, Epstein M, Paz O, Shimoni S, Caspi A. Head-up tilt table testing in syncope: safety and efficacy of isosorbide versus isoprotereno in pediatric population. Am Heart J 2008:156:477-82.

34. Delépine S, Prunier F, Lefthériotis G, Dupuis J, Vielle B, Geslin P, et al. Comparison between isoproterenol and nitroglycerin sensitized head-upright tilt in patients with unexplained syncope and negative or positive passive head-up tilt response. Am J Cardiol 2002;90:488-91.

35. Prabhu MA, Pillai V, Shenthar J. Comparison of efficacy, pattern of response, occurrence of arrhythmias, and the tolerability of nitroglycerine and isoprenaline as provocative drugs during head-up tilt test. Heart Lung Circ 2017:26:586-92.

36. Chu W, Wang C, Wu L, Lin F, Zou R. Oral rehydration salts: an effective choice for the treatment of children with vasovagal syncope. Pediatr Cardiol 2015;36:867-72.

37. Numata T, Abe H, Nagatomo T, Sonoda S, Kohshi K, Nakashima Y, et al. Successful treatment of malignant neurocardiogenic syncope with repeated tilt training program. Jpn Circ J 2000;64:406-9.

38. Reybrouck T, Heidbüchel $H$, Van de Werf $F$, Ector $H$. Tilt training: a treatment for malignant and recurrent neurocardiogenic syncope. Pacing Clin Electrophysiol 2000;23:493-8.

39. Krediet CT, van Dijk N, Linzer M, van Lieshout JJ, Wieling W. Maganement of vasovagal syncope: controlling or aborting faints by leg crossing and muscle tensing. Circulation 2002;106:1684-9.

40. Dehghan B, Sabri MR, Mansourian M. Nonpharmacologic treatments alone are enough to prevent the neurally mediated syncope: a 3 years follow-up study. Int J Prev Med. 2019;10:69.

41. Qingyou Z, Junbao D, Chaoshu T. The efficacy of midodrine hydrochloride in the treatment of children with vasovagal syncope. J Pediatr 2006;14:777-80

42. Liu XY, Wang C, Wu LJ, Hu CY, Lin MX, Cui XL, et al. Efficacy of midodrine hydrochloride in the treatment of children with vasovagal syncope. Zhonghua Yi Xue Za Zhi 2009;89:1951-54.

43. Salim MA, Di Sessa TG. Effectiveness of fludrocortisone and salt in preventing syncope recurrence in children: a double-blind, placebo-controlled, randomized trial. J Am Coll Cardiol 2005;45:484-8.

44. Scott WA, Pongiglione G, Bromberg BI, Schaffer MS, Deal BJ, Fish FA, et al. Randomized comparison of atenolo and fludrocortisone acetate in the treatment of pediatric neurally mediated syncope. Am J Cardiol 1995;76:400-2.

45. Müller G, Deal BJ, Strasburger JF, Benson DW Jr. Usefulness of metoprolol for unexplained syncope and positive response to tilt testing in young persons. Am J Cardiol 1993;71:592-5.

46. Zhang Q, Jin H, Wang L, Chen J, Tang C, Du J. Randomized comparison of metoprolol versus conventional treatment in preventing recurrence of vasovagal syncope in children and adolescents. Med Sci Monit 2008;14:199-203.

47. Kong Q, Yang X, Cai Z, Pan Y, Wang M, Liu M. Twenty-four-hour urine $\mathrm{NE}$ levels as a predictor of the therapeutic response to metoprolol in 
Arch Cardiol Mex. 2020;90(2)

children with recurrent vasovagal syncope. Ir J Med Sci 2019. [Epub ahead of print]. https://doi.org/10.1007/s11845-019-01979-9.

48. McLeod Ka, Wilson N, Hewitt J, Norrie J, Stephenson JB. Cardiac pacing for severe childhood neutrally mediated syncope with reflex anoxic seizures. Heart 1999;82:721-5

49. Kelly AM, Porter CJ, McGoon MD, Espinosa RE, Osborn MJ, Hayes DL. Breathholding spells associated with significant bradycardia: successful treatment with permanent pacemaker implantation. Pediatrics 2001; 108:698-702.
50. Suenaga $H$, Murakami M, Tani $T$, Saito $S$. Frequent neurally mediated reflex syncope in a young patient with dextrocardia: efficacy of catheter ablation of the superior vena cava-aorta ganglionated plexus. J Arrhythm 2015;31:172-6.

51. Debruyne P. "Cardio-neuromodulation" with a multielectrode irrigated catheter: a potential new approach for patients with cardio-inhibitory syncope. J Cardiovasc Electrophysiol 2016;27:1110-13.

52. Anderson JB, Willis $\mathrm{M}$, Lancaster $\mathrm{H}$, Leonard $\mathrm{K}$, Thomas $\mathrm{C}$. The evaluation and management of pediatric syncope. Peaditr Neurol 2016; 55:6-13. 